Review Article

\title{
Role of Hormones in the Control of Reproductive Physiology and Reproductive Behavior
}

\author{
Thi Mong Diep Nguyen \\ Faculty of Biology-Agricultural Engineering, Quy Nhon University, Quy Nhon City, Viet Nam \\ Email address: \\ nguyenthimongdiep@qnu.edu.vn
}

\section{To cite this article:}

Thi Mong Diep Nguyen. Role of Hormones in the Control of Reproductive Physiology and Reproductive Behavior. International Journal of Biomedical Materials Research. Vol. 7, No. 1, 2019, pp. 44-50. doi: 10.11648/j.jjbmr.20190701.16

Received: February 15, 2019; Accepted: March 22, 2019; Published: April 22, 2019

\begin{abstract}
In multicellular organisms, intercellular mediators such as hormones or growth factors and morphogens play primary roles in development and reproduction. Evolution of the signaling pathways in which these mediators are involved has thus played an important role in the appearance and success of these species. There are many reproductive modes and behaviours in the animal kingdom. A reproductive behaviour can be defined as all the actions taken by an organism toward the generation of one or more other organism that possesses at least some of its genetic patrimony. From a strict evolutionary point of view, the goal of an organism is to spread its genetic patrimony as much as possible in the next generations of its kind. This egoistic behaviour typically benefits for the entire species since the individuals spreading the most their genetic patrimony is generally those with the best genes and are thus helping their species to be the most fitted possible. In the present review, I briefly describe the implication of hormones in the control of mammals Reproduction and in their Sexual Behavior. In addition, since most multicellular organisms exhibit sexual reproduction, I also take into account hormonal control of reproduction together with sexual behavior.
\end{abstract}

Keywords: Hormones, HPG, GnRH, Gonadotropins, Reproduction, Sexual Behavior

\section{Introduction}

Hormones play a central role in the control of Reproductive Physiology and Reproductive Behavior. Reproduction itself is central to the Evolution of species [1]. Indeed, it is the slow drift of genotypes along successive generations of living species that gives rise to increasing variability of individuals, and occasio to speciation [2-6]. Speciation occurs when individuals from the same original species become unable to interbreed (and thus do not reproduce).

In the species with sexual reproduction, there is a need for male and female individuals to meet in order to allow sperm cells and oocytes to encounter and to fuse to produce a fertilized egg that will develop into a new individual. In animals, adapted behaviors are needed for the male and females to produce their gametes in a coordinated manner and to allow sperm cells to reach oocytes. Specific sexual behaviors have emerged that stimulate the attraction of fertile partners at optimal times and conditions for reproduction.
Clonal reproduction allows obtaining by simple cell division, two identical individuals from one in every generation; that is $2^{n}$ individuals in " $n$ " generations. There is no genetic admixture but transfers and genetic recombinations can intervene during each of the cell divisions and then may spread exponentially.

Sexual reproduction makes reference to the fusion, during fertilization, of gametes produced by male and female individuals. This gives a diploid cell, the fertilized egg called zygote, which is at the origin of all the cells of the new individual.

Sexual reproduction is numerically unfavorable with regard to clonal reproduction. However, it allows strong genetic admixture by crossing-over at the time of meiosis and so engenders a larger genetic variability. The bigger is the genetic diversity within a population and the slower will its speed of extinction be. This is because diversity permits some individuals to be fitted to new conditions; they survive and expand. Genetic variability is less important in asexual populations, because the "advantageous" mutations are less 
likely to occur at the same time in the same individual. On the other hand, in a sexual population, meiotic recombination allows to associate in a same individual mutation that appeared in different individuals. This allows fitted individuals to survive in new harsh conditions and even to conquer new environments.

Furthermore, genetic diversity allows better adaptation to parasites, because within the same population, there are sensitive and resistant individuals. Resistant individuals allow the preservation of the population. In the case of clonal reproduction, because the individuals are genetically identical, the population cannot survive if the genotype is sensitive to the parasites that are present.

\section{Hormones and Physiology of Reproduction}

\subsection{Hypothalamo-pituitary-gonadal (HPG) Axis}

The HPG axis is a complex system which allows an integrated regulation of reproduction in vertebrates (Figure 1).

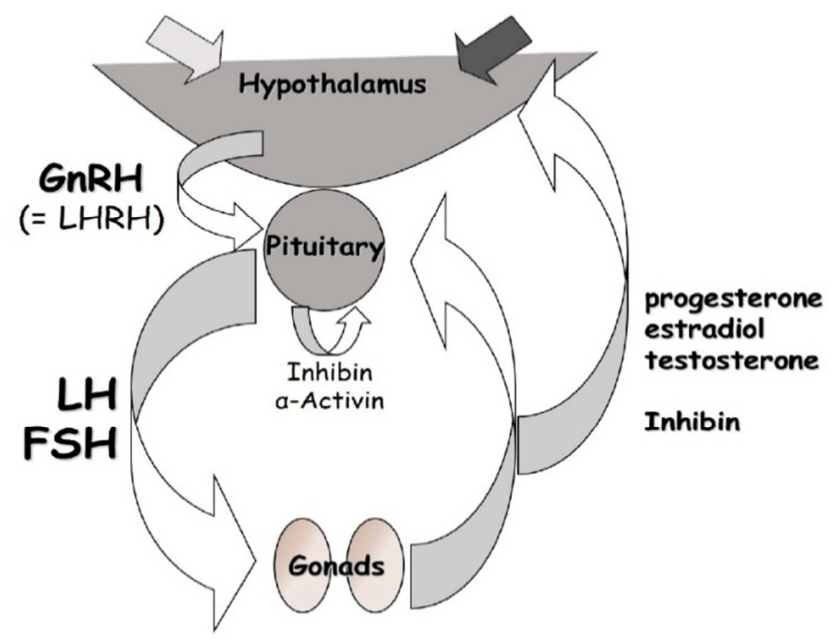

Figure 1. Diagram of the Hypothalamo-pituitary-gonadal (HPG) axis.

The hypothalamus, which is located at the basis of the brain, receives outcoming information as well as internal peripheral information. On the one hand, the hypothalamus indirectly receives physical abiotic information, such as variations of environmental light, temperature or pressure. These messages are integrated and interpreted in particular in seasonal species such as eels, sheep or horses for example. On the other hand, the hypothalamus perceives other biological external information, coming from congeners. For example, the male sexual partner emits pheromones that are perceived by the female in estrus. Also pheromones from the lamb permit its specific recognition by its mother. The peripheral internal information is mainly represented by gonadal hormones (sexual steroids, inhibin) which regulate the production and the secretion of hypothalamic GnRH (Gonadotropin Releasing Hormone) and consequently of the pituitary gonadotropins. The hypothalamus integrates these pieces of information and translates them into pulsatile secretion of
GnRH.

GnRH stimulates gonadotrope cells in anterior pituitary gland. This endocrine gland produces under stimulation by $\mathrm{GnRH}$, the pituitary gonadotrope hormones, LH (Lutenizing Hormone) and FSH (Follicle Stimulating Hormone). The pulsatility of GnRH together with gonadal steroids feedback, controls the differential secretion of FSH and LH. Gonadotropins structures are identical in males and females.

Gonads under FSH and LH stimulation produce sexual steroids (estradiol, progesterone, testosterone) which exert feedback effect on the hypothalamic-pituitary axis. These positive and negative regulations allow an effective control of the gametogenetic activity of gonads (production of oocytes and spermatozoa).

\subsection{GnRH Structure}

The gene encoding GnRH possesses 4 exons [8]. It is synthesized in the form of a precursor called preproGnRH (Figure 2). This preprohormone consists of a signal peptide, the hormone encoding sequence and a GAP (GnRH associated peptide). This precursor undergoes a maturation during which two proteolytic cuts take place. The signal peptide allows the addressing of the precursor towards the endoplasmic reticulum. During the axonal transport in GnRH neurons, GnRH maturation takes place in the secretion vesicles. In the endoplasmic reticulum, after removal of the signal peptide, the proGnRH is then proteolytically split into GnRH and GAP. The peptide GAP is co-secreted with GnRH but it is not known whether it exerts any physiological role. GnRH is a short peptide consisting of 10 amino acids. Its $\mathrm{N}$ - and C-terminal ends are modified: The N-terminal glutamine is cyclisized into pyro-Glu and the C-terminal glycine is modified into amide (CONH2). In this way, the GnRH peptide is protected from exopeptidases attacks at both ends.

GnRH exhibits a precise hairpin three-dimensional structure in which its $\mathrm{N}$-and $\mathrm{C}$-terminal residues are close together and form the specific binding site for GnRH receptor. The "Achilles' heel" of GnRH is the peptide bond Tyr (5)-Gly (6) which is easily split by endopeptidases. This fast degradation is important for the secretion pulsatility of GnRH. Indeed, its half-life must be short enough to allow its target cells to perceive its pulses.

One of the objectives of the pharmaceutical industry is to produce $\mathrm{GnRH}$ administrable in periphery that would not be degraded before arriving at the level of the pituitary gland. Thus it is necessary that this GnRH is more resistant to endopeptidases. The pharmaceutical industry synthesized GnRH superagonists. For example, the glycine in position 6 was replaced by diverse "exotic" amino acids (D-Lys, $\mathrm{a} D$-Ser-tert Butyl, D-leu, amine L-acid etc.). The proteolysis by the endopeptidases of the peptidic link after tyrosine 5 is no longer allowed and the active conformation of GnRH is thus preserved. These modified molecules are thus much more active than the native hormone because they are more stable in vivo thanks to their resistance to proteolytic enzymes. 


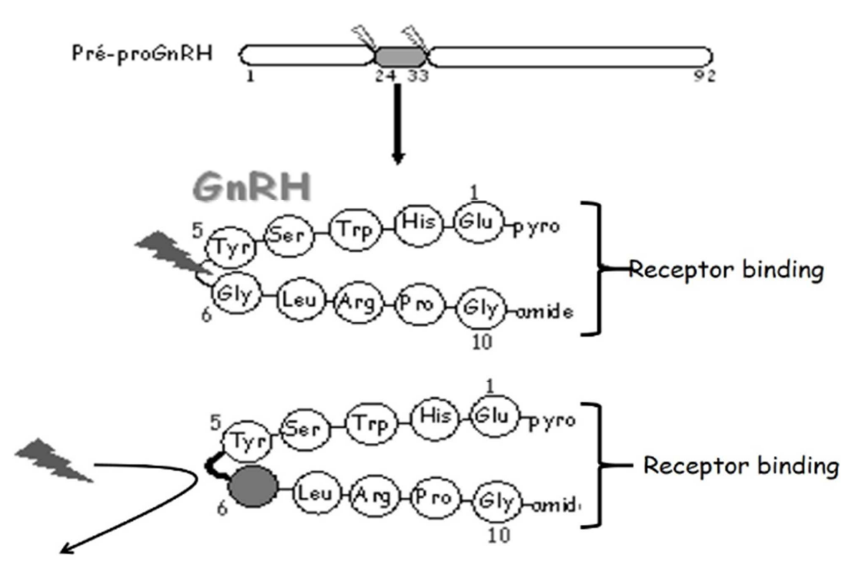

Figure 2. Biosynthesis, structure and degradation of natural GnRH and of a synthetic GnRH superagonist.

\subsection{Gonadotropins}

The gonadotropins are structurally much more complex than GnRH. The pituitary gonadotropins LH, FSH and the placental CG (chorionic gonadotropin present in Primates and Equidae) are heterodimers comprising one common $\alpha$-subunit and one specific $\beta$ subunit (Figure 3). These subunits are non-covalently bound together to form heterodimers. Indeed, treatments with urea, guanidinium, low or high $\mathrm{pH}$, or heat dissociate the heterodimer into its constituting subunits. This indicates that the subunits are non-covalently associated. Under separate form, the two subunits do not possess any biological activity. Only the $\alpha \beta$ heterodimers exert biological activity.

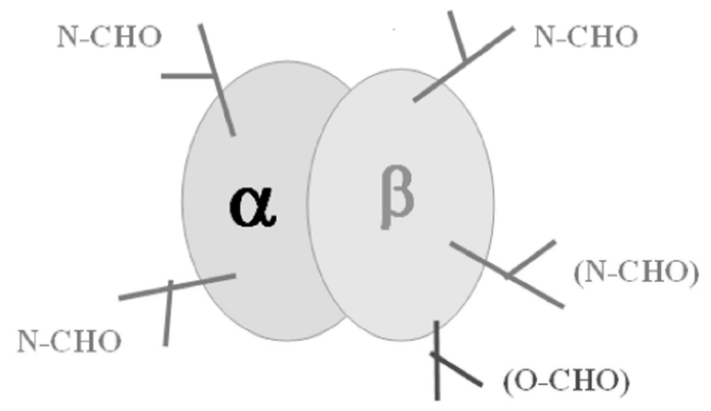

Figure 3. General simplified diagram of the glycoprotein hormones structure.

The $\alpha$-subunit is common to all GPHs as it is encoded by a unique gene (except in some fish) whereas the $\beta$-subunits are encoded by different genes and determine the biological specificity of the heterodimers.

The $\beta$ subunit possesses a "seat belt": The C-terminal extremity of $\beta$ subunit wraps around $\alpha$-subunit, then form an intra- $\beta$ disulfide bridge between cysteines $\beta 26$ and $\beta 110$. Therefore, although associated in a non-covalent way, $\alpha$ and $\beta$ subunits form a very stable heterodimer in physiological conditions.

Both subunits participate in receptor binding. The high affinity of gonadotropins towards their receptors is mainly governed by the $\alpha$-subunit whereas specificity is determined by the $\beta$-subunit, particularly by a "seat belt" portion.

Gonadotropins are glycoprotein hormones: they all possess three or four N-polysaccharide (N-CHO) chains, among whom two are borne by asparagines $(\mathrm{N})$ of $\alpha$-subunit and, according to hormones, one or two are borne by asparagines of $\beta$-subunits. Certain gonadotropins, essentially the placental gonadotropin $(\mathrm{CG})$, possess an additional C-terminal extension, called CTP, in their $\beta$-subunit beyond their seat belt sequence. This extension bears up to $4 \mathrm{O}$-saccharide chains on serines and threonines in hCG and up to 12 in the equine $\mathrm{CG}$ (eCG). The CTP with these O-saccharides plays an important role in the long half-life of these hormones in blood during gestation.

Although $\alpha$ and $\beta$ subunits are different, they nevertheless present a certain structural kinship. The phylogenetic analyses of these subunits' sequences highlight that they derive from a common molecular ancestor (Figure 4).

A few years ago, it was discovered in man, and then in the other vertebrates, the existence of genic sequences with strong homology with those of glycoprotein hormones (GPH) subunits. One with homology with $\alpha$-subunit was called A2 and the other one, with homology with $\beta$-subunit was called B5. The phylogenetic analysis of the sequences in available complete genomes in data banks showed that the A2 and B5 genes were present in vertebrates but also in very numerous metazoans, including the most ancient ones (-700 M years). The GPH subunits genes in vertebrates diverged from them more recently, shortly after or shortly before the emergence of vertebrates (-450 M years). Thus, it seems that the recently discovered $\mathrm{A} 2$ and $\mathrm{B} 5$ genes encode in fact extremely ancient molecules that subsequently were the roots for GPH subunits genes, which appeared at the time of vertebrates emergence. Nevertheless, A2 and B5 genes were conserved in all vertebrates indicating that they have not been functionally replaced by GPHs.

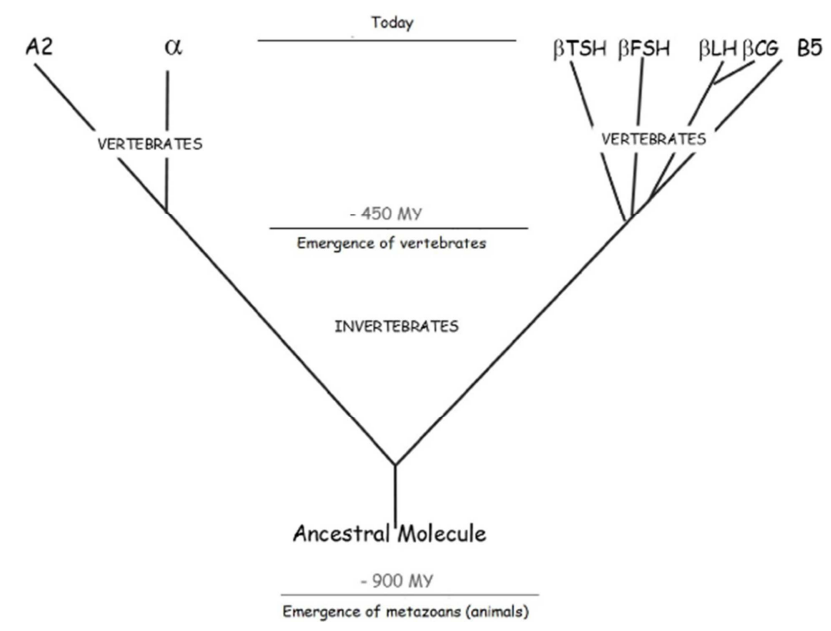

Figure 4. Evolution of $\alpha$ and $\beta$ subunits of glycoprotein hormones $(G P H)$ in vertebrates from their respective molecular ancestors $A 2$ and $B 5$ present in all metazoans (animals).

To understand the emergence of the pituitary gland and of gonadotropins in the central control of reproduction in vertebrates, we studied the presence of $\alpha$-subunit (A1) or of $\mathrm{A} 2$, and of LH $\beta$, FSH $\beta$, TSH $\beta$ and CG $\beta$ (B1-B4) or of B5 in taxons close to the appearance of vertebrates [9-10]. On one 
hand, the group of prochordates, including the amphioxus and the ascidian, that appeared just before the emergence of vertebrates and in which there is no pituitary gland and thus no gonadotropins. On the other hand, the groups of cyclostomes (agnathes) like the lamprey, and the primitive gnathostomes, like the eel, that appeared just after the emergence of vertebrates.

A2 and B5 glycoproteins have been found in the whole animal kingdom. It is the mark of an important role but this role has not been yet determined. There is disagreement among researchers, concerning the ability of A2 and B5 to form heterodimers like GPH $\alpha$ - and $\beta$-subunits do. They may rather act without association but maybe not independently on a functional basis. Indeed, they are always either both present (in a large majority of animal species) or both absent (hymenopters) [11].

\subsection{GnRH and Gonadotropins Receptors}

$\mathrm{GnRH}$ and gonadotropins are structurally very different molecules but their receptors belong to the same family, i.e. that of the seven transmembrane domains receptors (7TMR), coupled to trimeric G proteins (GPCR) (Figure 5). The transmembrane domains are hydrophobic and amino acids situated between these domains form three extracellular loops and three intracellular loops.

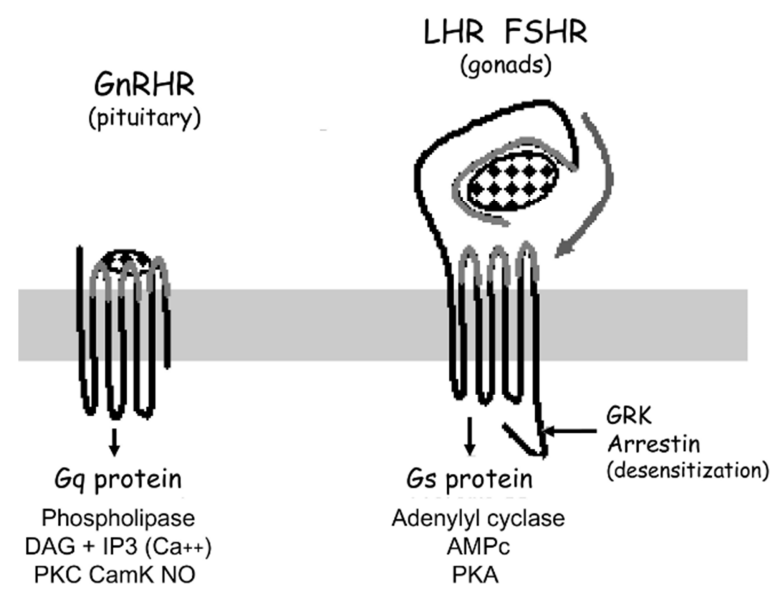

Figure 5. Simplified diagram of the structures of the receptors for GnRH, FSH and LH. All are receptors with 7-TM receptors coupled with heterotrimeric $(\alpha \beta \gamma)$ G proteins (GPCR).

\section{* GnRH Receptor}

The GnRH receptor (GnRHR) is located at the level of the adenohypophysis (anterior pituitary gland) [12]. GnRH binds to extracellular loops of the GnRHR. This binding leads to a change in the conformation of the intracellular parts of the receptor, which then allows a direct coupling with the $\mathrm{Gq}$ protein. This $\mathrm{Gq}$ protein activates a specific effector: phospholipase C (PLC), which leads to synthesis of inositol triphosphate (IP3) and of diacylglycerol (DAG). Together, IP3 and DAG are responsible for the mobilization of the intracellular calcium from endoplasmic reticulum to cytoplasm, and for the activation of protein kinase $\mathrm{C}$ (PKC). GnRH secretion is pulsatile and the pulsatility frequency influences $\mathrm{FSH} / \mathrm{LH}$ secretion ratio by pituitary gonadotropes [13].

\section{* LH and FSH receptors}

LHR and FSHR are essentially present at the level of gonads. LH and FSH bind to the extracellular domain of their respective receptors (LHR and FSHR). The complex formed by the receptor extracellular domain and hormone interacts with the 7-span transmembrane part of the receptor and triggers a change in the relative orientations of the transmembrane $\alpha$-helixes. This leads to a change in the conformation of the receptor's intracellular loops, allowing a direct coupling with Gs protein, the $\alpha$-subunit of which subsequently interacts with adenylate cyclase (AC) and stimulates it. Activated adenylate cyclase catalyzes the synthesis of cyclic AMP from ATP. Cyclic AMP is an intracellular second messenger, which activates protein kinase A (PKA).

The LHR and FSHR, in contrast to GnRHR, possess an intracellular C-terminal extremity. This region can be phosphorylated by a specific kinase (GRK) recruited by the $G$ protein $\beta \gamma$ subunits. This phosphorylation allows the subsequent recruitment of arrestin which, as its name indicates, is responsible for the arrest of the signalling pathway. This mechanism promotes desensitization of cells to the hormone. Arrestin is also a scaffolding protein that recruits partners of the MAPK cascade and stimulates this pathway [14].

\subsection{Nuclear Receptors for Steroid Hormones}

Steroid hormones penetrate into cells and bind to specific receptors exercising their action at the nuclear level. These receptors are transcription factors that are regulated by binding with their specific hormonal ligand [15]. They possess a typical structure constituted by several functional domains (Figure 6).
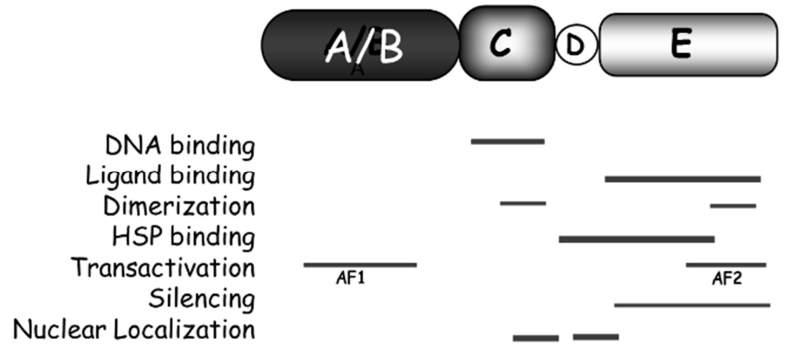

Figure 6. General diagram of nuclear receptors structure showing functional domains.

(1) The A/B domain is a ligand-independent transcription activation domain (Activation Function-1 [AF-1]). It is located at the N-terminal end of the protein. It is a site of interaction with general transcription factors, through CBP (Creb Binding Protein). CBP stabilizes the complex formed by general transcription factors at the initiation site of transcription and allows recruitment of type II RNA polymerase which then transcribes the coding sequence of the gene into messenger RNA.

(2) The C domain is responsible for receptor binding to 
DNA. It consists of two structures called "zinc fingers". The nuclear receptors bind to DNA as dimers recognized by palindromic DNA sequences called HRE (Hormone Responsive Elements). These short sequences are usually situated in the promoter region of hormones target genes.

(3) A hinge region or D domain follows the DNA-binding domain DNA. This domain gets flexibility to the protein allowing interactions between the other domains.

(4) The E domain is the ligand-binding domain. It is located at the C-terminal end of the receptors. Besides, the ligand-binding $\mathrm{E}$ domain also bears a ligand dependent transcription region (Activation Function-2 [AF-2]), as well as sequences of interaction with heat-shock proteins (HSPs), dimerization sequences and nuclear localization sequences.

\subsection{At Physiological Level}

We saw previously that GnRH secretion is pulsatile. The pulsatility frequency influences pituitary responses (LH or FSH). How is GnRH secretion pulsatility controlled?

GnRH neurons are not numerous (1000 - 3000 in Human) and scattered in the hypothalamus. Their activity is controlled by either the other GnRH neurons forming a network, or neurons producing other neurotransmitters. These observations were made during studies on the regulation of GnRH neurons by estradiol. GnRH neurons do not express estradiol receptors whereas other neurons do (GABA, NA, Kiss). Thus estradiol acts indirectly on GnRH neurons through a network of other types of neurons.

Besides, photoperiod (duration of nights and days) is translated into waves of melatonin secretion in the brain. Melatonin acts on dopamine neurons and then, dopamine inhibits GnRH secretion by GnRH neurons [16]. Consequently, it inhibits LH and FSH secretion. This explains how photoperiod regulates reproductive function in seasonal species [17].

LH and FSH exhibit different secretion profiles. In response, gonads secrete sexual steroids and molecules such as activin, inhibin and the BMP (Bone Morphogenetic Proteins) which exert feed back control at the level of the pituitary gland. Sexual steroids have an influence on the primary and secondary sexual characters, as well as on other physiological functions like growth.

Beside gonadotropins and sexual steroids, which are directly involved in reproduction control in vertebrates, there are numerous hormones that, indirectly, affect reproductive function.

First, the adipose tissue mass has a disfavourable influence on reproduction. This primarily occurs via the secretion of adipokines, such as leptin and adiponectin but also resistin, visfatin, chemerin etc. Leptin, which is the main "satiety hormone" acts on the hypothalamus via pro-opiomelanocortin (POMC), neuropeptide Y (NPY), and agouti-related peptide (AgRP) neurons to translate at the central nervous system, acute changes in nutrition and energy expenditure. These neurons interact with GnRH and Kiss neurons and modify GnRH secretion and therefore LH and FSH secretion by the pituitary. Adiponectin mediates insulin sensitivity at many levels of the reproductive axis [18].

A number of the numerous hydrophobic small molecules produced by chemical industry (paints, drugs, pesticides, plastics, etc.) are able to fit to the binding pocket of nuclear receptors and to either stimulate or inhibit their transcription factor activity. When present in the environment, they can interfere with the endocrine network and can thus alter the proper regulation of physiological functions, in particular of Reproduction. These molecules are collectively designated as "endocrine disruptors".

For example, bisphenol-A (BPA) that is a molecule used in the synthesis of plastics, has been found to bind to the estradiol receptor (ER). It exhibits a 100 000-fold lower affinity than estradiol towards ER but because of its presence in alimentary plastics, in particular in bottles for babies (who are very sensitive targets), it has been banned in many countries.

\section{Hormones and Behavior of Reproduction}

Sexual reproduction requires that males and females express the necessary behavior in order to permit meeting of their gametes and fertilization. Sexual gonadal steroids play essential roles in this behavior.

\subsection{Experimental Aspects}

Sexual steroids have two levels of action; on the one hand during fetal development and on the other hand during sexual life at adulthood:

During fetal development

In the newborn child, sexual steroids have organizing effects, in particular for brain sexualization during prenatal and/or perinatal periods. Injection of testosterone in a newborn female rat abolishes its capacity to present the lordosis reflex during adulthood. It has thus a permanent effect.

In adults, sexual steroids have reversible activating effects. For example, when castrated a male rat loses its copulation behavior. Injection of testosterone restores its copulatory activity but it quickly disappears when the injections stop.

How does the brain become sexualized? Can we say that the testosterone is the male hormone and the estradiol the female hormone?

In the brain, testosterone is transformed into estradiol by the enzyme aromatase. In rat, estradiol is responsible for brain masculinization and defeminization. Defeminization is measured in adult females by the loss of their lordosis reflex whereas masculinization is measured in adult castrated males through their re-acquisition of copulation behavior.

Nevertheless, females produce estradiol at puberty. Why does not ovarian estradiol masculinize females' behavior?

When young naive wild-type $(+/+)$ female mice are put in the presence of active adult males, the number of lordoses 
quickly increases with the repetition of tests.

When AFP -/- (invalidation of the alpha-fetoprotein gene) mice are subjected to the same test, they present no lordosis reflex, indicating that they are defeminized. What role plays AFP?

In males, testosterone enters into the brain where it is metabolized into estradiol by aromatase. The brain is thus masculinized by estradiol impregnation. In females, circulating estradiol is bound to alpha-fetoprotein [19] and cannot penetrate into the brain. Their brain is feminized indicating that the female behavior would be the default behavior. Alpha-fetoprotein is also present in males, but it is not able to bind testosterone and does not impede its entry into the brain.

The action of the testosterone is mainly indirect in the brain as well as in peripheral target organs (seminal vesicles, prostate, epididymis, etc.). In the brain, it is metabolized by aromatase into estradiol and acts via the estrogen receptors (Figure 7). At the peripheral level, it is transformed by the $5 \alpha$-reductase into DHT, which has a stronger affinity than testosterone towards androgen receptors. Interestingly, aromatase activity in the male brain is regulated in time domains corresponding to the slow "genomic" and faster "nongenomic" modes of action of oestrogens [20]. These actions correspond to actions mediated by the classical nuclear receptors and membrane estrogen receptors respectively.

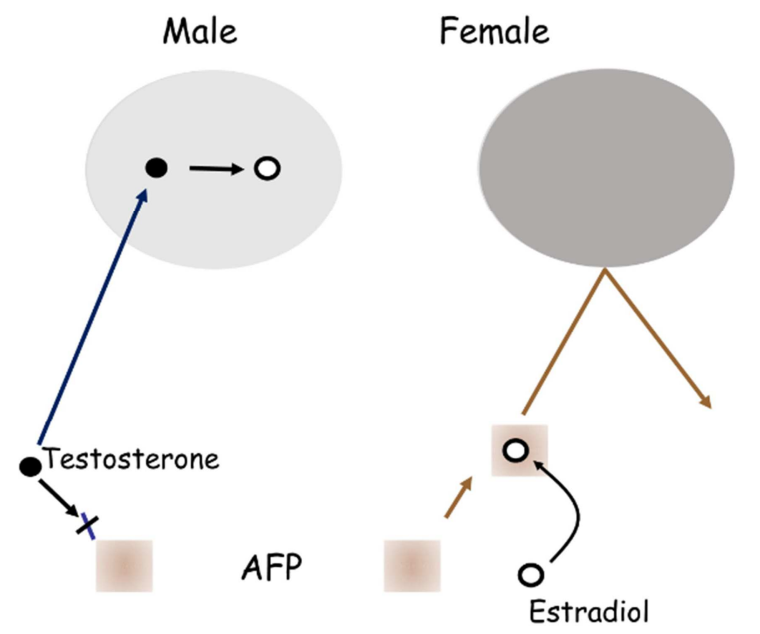

Figure 7. How does estradiol masculinize the brain of male rats but not that of females (AFP: alpha-fetoprotein).

At adulthood

Messenger RNA and protein neo-synthesis are required for the estrogenic regulation of lordosis behavior in adult female rodents. At least part of the behavioral effect of estrogen occur through epigenetic modification, particularly by histone modification [21]. These modifications affect the expression of various genes, among them the progesterone receptor and oxytocin receptor at different levels in the hypothalamus.

Pheromones, that are kind of inter-individual hormones, play a prominent role in sexual behavior. In mammals, they are detected by receptors in the vomeronasal organ [22] and trigger partners arousal and sexual intercourse. In insects, the pheromone receptors are located in different positions, generally in the antennas, and permit long-distance communication allowing females to attract competing males.

\subsection{Clinical Aspects}

How does that take place in the human species? Pathological cases allow to determine whether it is testosterone or estradiol that masculinizes the brain:

Congenital adrenal hyperplasia:

In XX genotype foetus, the fetal adrenal gland produces testosterone. In 1 case over 2000, the testosterone concentration is sufficient to masculinize the brain [23-24.]. At adulthood, these women present mainly a heterosexual orientation, but the percentage with a homosexual orientation seems higher than in the control population.

Syndrome of complete insensitivity to androgens (AR-/-):

In this syndrome, androgen receptors (AR) are non-functional. The genetically XY individuals have testicles, but often a feminine external phenotype as well as a generally feminine behavior.

These pathological cases suggest that it is testosterone itself which would masculinize the human brain (and not estradiol as in rodents). But one must be careful with this conclusion, because of the weight of education and social identification. Indeed, these genetically XY male individuals have a feminine aspect, thus are educated as girls and consequently become identified and behave as girls. The weight of the culture and social relationships in the human species render difficult to determine precisely the role of hormones in sexual behavior.

\section{Conclusions}

In the present article, I have summarized the most important aspects of hormonal regulations of Reproduction and of sexual behavior. Due to the limited space of a paper, I have focused on mammalian reproduction and essentially, on the human species. Hormones and related molecules such as growth factors, morphogens and cytokines play primary roles in the development of multicellular organisms.

\section{References}

[1] Darwin, C. On the Origin of Species by Means of Natural Selection, 1st edition; 1859 (London: John Murray).

[2] Blacher, P.; Huggins, T. J.; Bourke, A. F. G. Evolution of ageing, costs of reproduction and the fecundity-longevity trade-off in eusocial insects. Proc Biol Sci 2017, 284, doi:10.1098/rspb.2017.0380.

[3] Brooks, R. C.; Garratt, M. G. Life history evolution, reproduction, and the origins of sex-dependent aging and longevity. Ann N Y Acad Sci 2017, 1389, 92-107, doi: $10.1111 /$ nyas. 13302 .

[4] Crouch, D. J. M. Statistical aspects of evolution under natural selection, with implications for the advantage of sexual reproduction. $J$ Theor Biol 2017, 431, 79-86, doi:10.1016/j.jtbi.2017.07.021. 
[5] Kolodny, O.; Stern, C. Evolution of risk preference is determined by reproduction dynamics, life history, and population size. Sci Rep 2017, 7, 9364, doi:10.1038/s41598-017-06574-5.

[6] Olejarz, J.; Veller, C.; Nowak, M. A. The evolution of queen control over worker reproduction in the social Hymenoptera. Ecol Evol 2017, 7, 8427-8441, doi:10.1002/ece3.3324.

[7] Kraus, C.; Schiffer, P. H.; Kagoshima, H.; Hiraki, H.; Vogt, T.; Kroiher, M.; Kohara, Y.; Schierenberg, E. Differences in the genetic control of early egg development and reproduction between C. elegans and its parthenogenetic relative D. coronatus. Evodevo 2017, 8, 16, doi:10.1186/s13227-017-0081-y.

[8] Tostivint, H. Evolution of the gonadotropin-releasing hormone $(\mathrm{GnRH})$ gene family in relation to vertebrate tetraploidizations. Gen Comp Endocrinol 2011, 170, 575-581, doi:10.1016/j.ygcen.2010.11.017.

[9] Alvarez, E.; Cahoreau, C.; Combarnous, Y. Comparative structure analyses of cystine knot-containing molecules with eight aminoacyl ring including glycoprotein hormones (GPH) alpha and beta subunits and GPH-related A2 (GPA2) and B5 (GPB5) molecules. Reprod Biol Endocrinol 2009, 7, 90, doi:10.1186/1477-7827-7-90.

[10] Cahoreau, C.; Klett, D.; Combarnous, Y. Structure-function relationships of glycoprotein hormones and their subunits' ancestors. Front Endocrinol (Lausanne) 2015, 6, 26, doi:10.3389/fendo.2015.00026.

[11] Dos Santos, S.; Mazan, S.; Venkatesh, B.; Cohen-Tannoudji, J.; Querat, B. Emergence and evolution of the glycoprotein hormone and neurotrophin gene families in vertebrates. $B M C$ Evol Biol 2011, 11, 332, doi:10.1186/1471-2148-11-332.

[12] Mo, Y.; Peng, P.; Zhou, R.; He, Z.; Huang, L.; Yang, D. Regulation of gonadotropin-releasing hormone (GnRH) receptor-I expression in the pituitary and ovary by a $\mathrm{GnRH}$ agonist and antagonist. Reprod Sci 2010, 17, 68-77, doi:10.1177/1933719109348026.

[13] Armstrong, S. P.; Caunt, C. J.; Fowkes, R. C.; Tsaneva-Atanasova, K.; McArdle, C. A. Pulsatile and sustained gonadotropin-releasing hormone $(\mathrm{GnRH})$ receptor signaling: does the ERK signaling pathway decode GnRH pulse frequency? J Biol Chem 2010, 285, 24360-24371, doi:10.1074/jbc.M110.115964.

[14] Reiter, E.; Ahn, S.; Shukla, A. K.; Lefkowitz, R. J. Molecular mechanism of beta-arrestin-biased agonism at seven-transmembrane receptors. Annu Rev Pharmacol Toxicol 2012, 52, 179-197, doi:10.1146/annurev.pharmtox.010909.105800.
[15] Gustafsson, J. A. Historical overview of nuclear receptors. $J$ Steroid Biochem Mol Biol 2016, 157, 3-6, doi:10.1016/j.jsbmb.2015.03.004.

[16] Badruzzaman, M.; Bapary, M. A.; Takemura, A. Possible roles of photoperiod and melatonin in reproductive activity via changes in dopaminergic activity in the brain of a tropical damselfish, Chrysiptera cyanea. Gen Comp Endocrinol 2013, 194, 240-247, doi:10.1016/j.ygcen.2013.09.012.

[17] Abecia, J. A.; Chemineau, P.; Gomez, A.; Keller, M.; Forcada, F.; Delgadillo, J. A. Presence of photoperiod-melatonin-induced, sexually-activated rams in spring advances puberty in autumn-born ewe lambs. Anim Reprod Sci 2016, 170, 114-120, doi:10.1016/j.anireprosci.2016.04.011.

[18] Rak, A.; Mellouk, N.; Froment, P.; Dupont, J. Adiponectin and resistin: potential metabolic signals affecting hypothalamo-pituitary gonadal axis in females and males of different species. Reproduction 2017, 153, R215-R226, doi:10.1530/REP-17-0002.

[19] VanBrocklin, H. F.; Brodack, J. W.; Mathias, C. J.; Welch, M. J.; Katzenellenbogen, J. A.; Keenan, J. F.; Mizejewski, G. J. Binding of 16 alpha-[18F]fluoro-17 beta-estradiol to alphafetoprotein in Sprague-Dawley female rats affects blood levels. Int J Rad Appl Instrum B 1990, 17, 769-773.

[20] de Bournonville, C.; Ball, G. F.; Balthazart, J.; Cornil, C. A. Rapid changes in brain aromatase activity in the female quail brain following expression of sexual behaviour. $J$ Neuroendocrinol 2017, 29, doi:10.1111/jne.12542.

[21] Forger, N. G. Epigenetic mechanisms in sexual differentiation of the brain and behaviour. Philos Trans R Soc Lond B Biol Sci 2016, 371, 20150114, doi:10.1098/rstb.2015.0114.

[22] Morozova, S. V.; Savvateeva, D. M.; Svistushkin, V. M.; Toporkova, L. A. [The role of the vomeronasal system in the formation of the human sexual behaviour]. Vestn $\begin{array}{llll}\text { Otorinolaringol } & 2017, & 82, & 90-94,\end{array}$ doi:10.17116/otorino201782190-94.

[23] Tanaka, M.; Enatsu, N.; Chiba, K.; Fujisawa, M. Two cases of reversible male infertility due to congenital adrenal hyperplasia combined with testicular adrenal rest tumor. Reprod Med Biol 2018, 17, 93-97, doi:10.1002/rmb2.12068.

[24] Berenbaum, S. A.; Beltz, A. M.; Bryk, K.; McHale, S. Gendered Peer Involvement in Girls with Congenital Adrenal Hyperplasia: Effects of Prenatal Androgens, Gendered Activities, and Gender Cognitions. Arch Sex Behav 2018, 47, 915-929, doi:10.1007/s10508-017-1112-4. 